\title{
Indirect choroidal ruptures: aetiological factors, patterns of ocular damage, and final visual outcome
}

\author{
Christopher Mark Wood, John Richardson
}

\begin{abstract}
Indirect choroidal ruptures result from blunt ocular trauma and have a pathognomonic fundal appearance. We analysed a group of 30 patients with indirect choroidal ruptures with specific reference to the circumstances of the injury, the pattern of ocular damage, the cause of any visual loss, and the final visual outcome. Using this analysis we deduce a pathogenetic explanation for the characteristic fundus signs in patients with indirect choroidal ruptures. The majority of cases were young males injured during sport or by an assault, a minority were injured at work. Diffuse nonfocal impact injuries due to punches were associated with ruptures concentric with and adjacent to the optic disc. Focal impact injuries, due to projectiles, showed more extensive ocular damage. Seventeen of $\mathbf{3 0}$ patients regained $6 / 12$ vision after injury. Injuries due to projectiles and temporally situated ruptures were associated with a poorer visual outcome than others. Macular damage was the commonest cause of visual loss, principally due to pigmentary maculopathy, traumatic inner retinal damage, and choroidal neovascular membranes rather than direct foval damage by the rupture.
\end{abstract}

The typical appearance in the fundus of indirect choroidal ruptures is well documented..$^{1-3}$ Little attempt has been made to explain the mechanism by which peripapillary ocular damage results from closed trauma to the anterior segment or to relate the clinical appearance of indirect choroidal ruptures with the type of injury sustained and the final visual outcome.

We report here our studies on a group of patients with indirect choroidal ruptures, noting the circumstances of the injury, the extent of ocular damage, cause of any visual loss, and longterm visual outcome. We suggest a working hypothesis to account for the mechanism of production of indirect choroidal ruptures and pose the question of a possible role of minor trauma in presumed non-traumatic maculopathies.

\section{Materials and method}

Sunderland Eye Infirmary, Queen Alexandra Road, Sunderland, Tyne and Wear SR2 9HP C M Wood

J Richardson

Correspondence to: C Wood, FRCS Ed.

Accepted for publication 5 October 1989 studied ( 27 males and three females). The patients were either followed up prospectively from the time of the injury (seven cases) or were already being followed up (16 cases), or they were identified as having an indirect choroidal rupture from our photographic files and asked to attend for further examination (seven cases).
The average age at the time of the injury was 24 years (range 8-45 years). The average follow-up time after injury was $4 \frac{1}{2} 2$ years (range 6 months to 41 years, though in only two cases had the injury occurred more than 10 years previously).

All the patients gave a detailed history of the original injury and then had a full ophthalmic assessment - including computerised visual fields, retinal photography, fluorescein angiography, electroretinography, and measurement of pattern reversal visual evoked potentials.

Individual fundal photographs were projected on to a screen, enabling the outlines of each rupture to be marked. By this method composite patterns of rupture following different types of injury were produced. For ease of comparison all slides were projected to produce the image of a left fundus - that is, photographs of right fundal lesions were inverted.

\section{Results}

From the patients' histories we could identify three groups of circumstances in which the injury occurred - namely, sport, assaults, and accidents at work. Eight of 11 injuries due to sport occurred in males aged 11-15 years, and in $9 / 11$ cases the left eye was injured $(0.05>$ $p>0.02)$. Nine of 13 of the injuries following assaults occurred in males aged 15-31 years; in 8/ 13 cases the left eye was injured (not a significant difference). Accidents at work accounted for six cases, four of these patients being over 30 years old; in 2/6 cases the left eye was affected (not a significant difference).

The type of injury was divisible into two groups - those due to punches, where the eye was subjected to a diffuse force, and those due to projectiles, which had a more focal impact on the globe. The distribution of ruptures in seven eyes subjected to punches is shown in Figure 1 and can be seen to be mainly restricted to the peripapillary region. These ruptures were

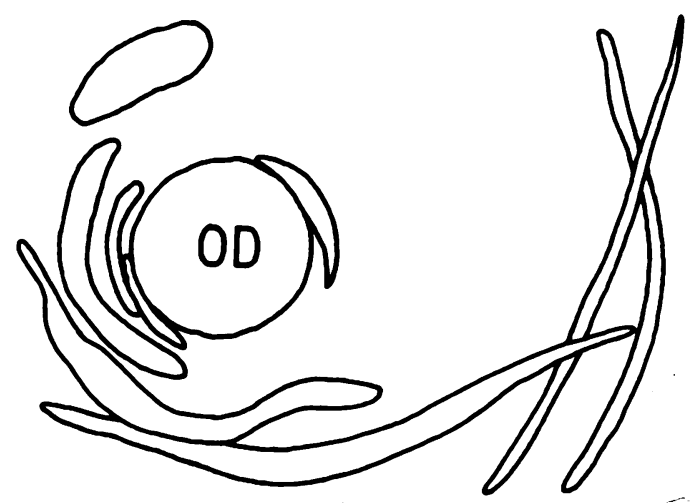

Figure 1: Composite diagram illustrating the sites of indirect choroidal rupture in eyes suffering injuries due to punches. 


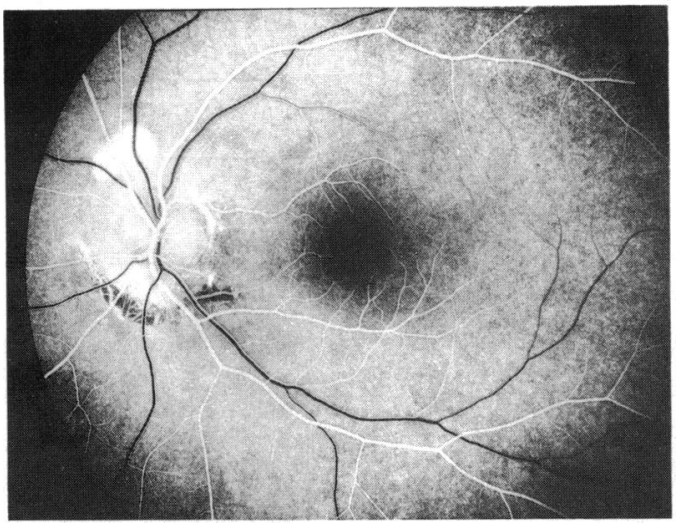

Figure 2: Indirect choroidal rupture inferior to the disc following a punch. Fluorescein angiogram shows a partial thickness rupture with smooth curved appearance.

typically curved or curvilinear in appearance, concentric with, and usually inferiorly or temporally to, the disc (Fig 2). Anterior uveal injuries were minimal, with only one case of significant angle recession and no cases of direct choroidal ruptures (Table I).

Indirect choroidal ruptures resulting from injuries due to various projectiles, hand held implements, or kicks (Table II) striking the eye are shown in Figure 3. These indirect choroidal ruptures were less likely to occur near the disc (Fig 4) and more likely to be multiple, extensive, and irregular. The associated anterior uveal injuries were also more severe (Table I), sometimes indicating the site of impact. Five patients had a continuous path of damage extending from the anterior uvea as a direct choroidal rupture or iridodialysis, through areas of retinal pigment epithelium damage, to indirect choroidal ruptures at the posterior pole. This more extensive pattern of ocular damage reflects the more severe nature of the injury due to the higher kinetic energy and focal impact on the globe.

The majority of patients (17/30) regained vision of $6 / 12$ after the injury. Temporally situated ruptures were associated with a poorer visual outcome (Table III), as macular damage was more likely to occur. In five eyes ruptures involving the fovea appeared to be directly responsible for a reduction in vision. However, in a further 10 eyes with macular damage the

TABLE I Characteristics of indirect choroidal ruptures related to type of injury sustained. Included in the projectile group are kicks and hand held weapons such as pickaxe handles

\begin{tabular}{lllll}
\hline & & $\begin{array}{l}\text { Punches } \\
(n=7)\end{array}$ & $\begin{array}{l}\text { Projectiles } \\
(n=23)\end{array}$ & $\begin{array}{l}\text { Overall } \\
(n=30)\end{array}$ \\
\hline Site & Peripapillary & 7 & 13 & 20 \\
\multirow{4}{*}{ Number } & Peripheral & 0 & 10 & 10 \\
\multirow{4}{*}{ Appearance } & Single & 4 & 9 & 13 \\
& Multiple & 3 & 14 & 17 \\
Anterior uveal injury & Irregular & 7 & 17 & 24 \\
\end{tabular}

TABLE II Types of projectiles or weapons responsible for focal impact injuries to the eye

\begin{tabular}{ll}
\hline Arrow/stick & 8 \\
Metal & 5 \\
Stones/brick & 4 \\
Plastic & 2 \\
Squash ball & 2 \\
Kick & 2 \\
\hline
\end{tabular}

pigmentary mot directly involve the fove retinal damage (two eyes) were responsible the loss of vision. There were two cases wher thin partial thickness rupture passed very close to the fovea (Fig 5), yet the patients retained good central vision. Patients suffering an injury as a result of a punch retained a better level of visual acuity than patients suffering an injury due to a projectile (Table IV).

Visual field defects noted corresponded to areas of retinal pigment epithelium damage visible ophthalmoscopically rather than to the individual ruptures, and there were no nerve fibre bundle scotomata related specifically to an indirect choroidal rupture. There were three patients with nerve fibre bundle field defects which did not correspond to the chorioretinal injury and one patient with an altitudinal field defect. In these four cases we considered that the clinical appearance of the optic disc (haemorrhage on the disc in the acute stage, or pallor out of proportion with the fundal changes) and the field defect together with abnormal visually evoked responses suggested the presence of a primary optic nerve injury in addition to the chorioretinal damage.

Electrophysiological assessment generally reflected the fundal changes, patients with extensive retinal pigment epithelium changes having abnormal electroretinograms. As the macular region dominates the pattern visually evoked potential (VEP), delay in the response or a reduction in amplitude was seen in patients with overt macular damage. Two patients with apparently healthy maculae had VEPs with delay or reduced amplitude due to the presence of an optic nerve injury. A further two patients with extensive field defects and optic atrophy as well as macular damage had grossly abnormal VEPs due to the combination of macular and optic nerve injuries.

Delayed complications may occur after concussional eye injuries, ${ }^{2-4}$ leading to further (or late) visual loss, and $8 / 30$ of our series developed changes which could have led to a further deterioration in visual function (one patient with glaucoma, two with retinal detachments, five developing subretinal neovascular membranes).

\section{Discussion}

Eye injuries most commonly affect young males and represent a significant cause of visual loss. ${ }^{56}$ Concussional injuries result from the direct effect of trauma at the site of impact or from the effect of transmitted force. ${ }^{+}$Energy can be transmitted across the globe either through the vitreous gel (contrecoup theory) ${ }^{7}$ or via the walls of the globe. ${ }^{8}$ However, the contrecoup theory cannot account for the path of damage from impact site to posterior pole which we observed in some of our patients. In addition, since the eye is most vulnerable to injury from an inferotemporal direction, ${ }^{9}$ then injuring forces passing directly through the vitreous (as in the contrecoup theory) could be expected to produce choroidal ruptures on the nasal side of the disc, whereas in fact most are located on the temporal side. Experimental work on pigs' eyes suggests that the transmission of energy in concussional eye injuries occurs via the walls of the globe ${ }^{8}$ and has been unable to demonstrate evidence of contrecoup injury. ${ }^{10}$ To our knowledge no experimental study has been able to demonstrate convincingly the existence of contrecoup forces in the pathogenesis of concussional eye injuries. Thus the contrecoup mechanism may explain 


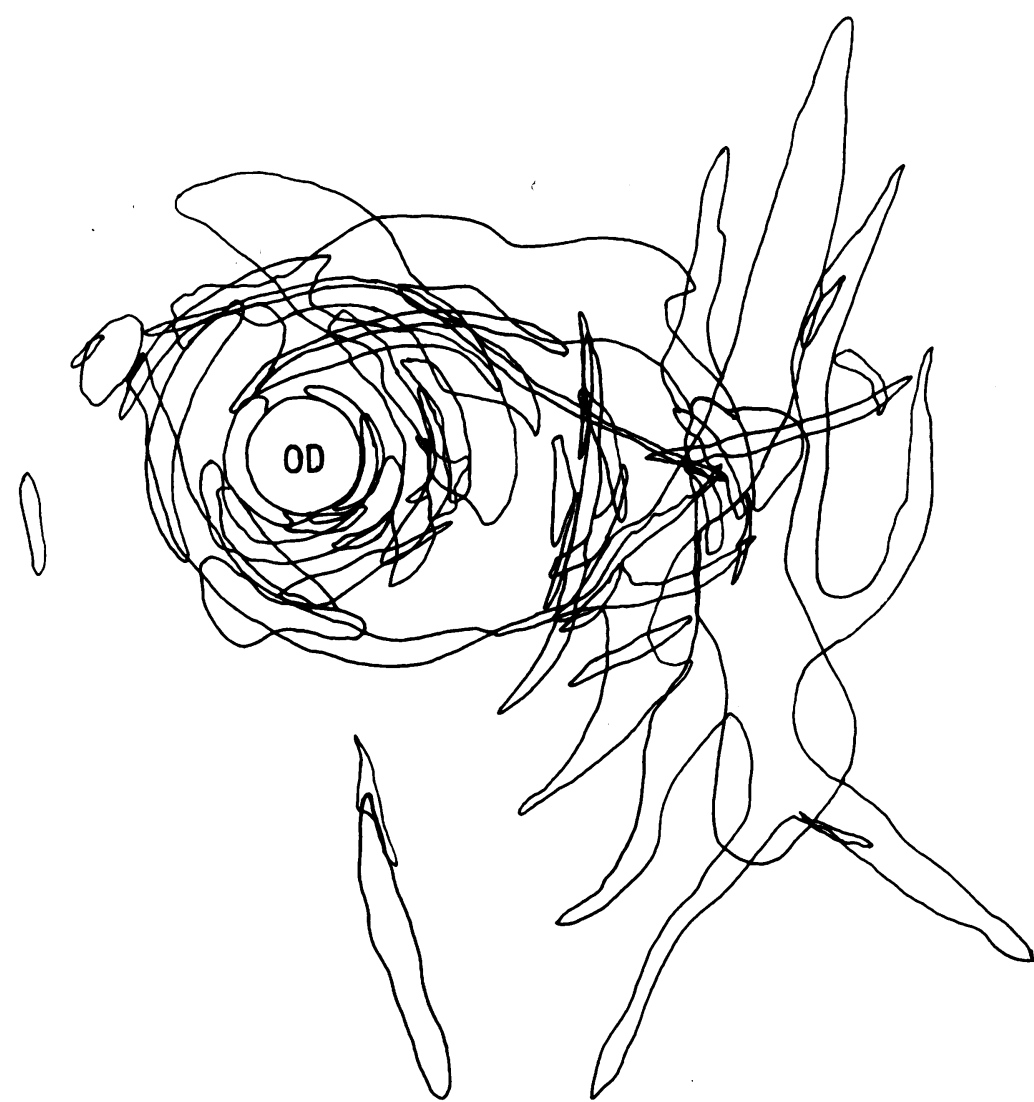

Figure 3: Composite diagram illustrating the sites of indirect choroidal ruptures in eyes suffering injuries from projectiles, various weapons, and kicks.

damage to movable structures within a rigid sphere such as the skull but does not account for the pattern of damage in a resilient, deformable sphere such as the globe.

When energy is conducted through the walls of a sphere, any point at which there is a discontinuity in the wall, or an attachment to the wall, will cause a localised increase in stress. Consequently the attachments at the vitreous base will produce localised increase in stress in the peripheral retina. Similarly the discontinuity produced in the wall of the globe by the entry of the optic nerve and posterior ciliary arteries, together with the effect of external attachments to the wall of the globe (the optic nerve, posterior ciliary vessels and the inferior oblique, and the internal attachment of the posterior vitreous) will induce an increase in stress in the peripapillary retina and choroid. " The overall effect is that acute deformation maximises stress at the peripheral retina and the peripapillary retina and

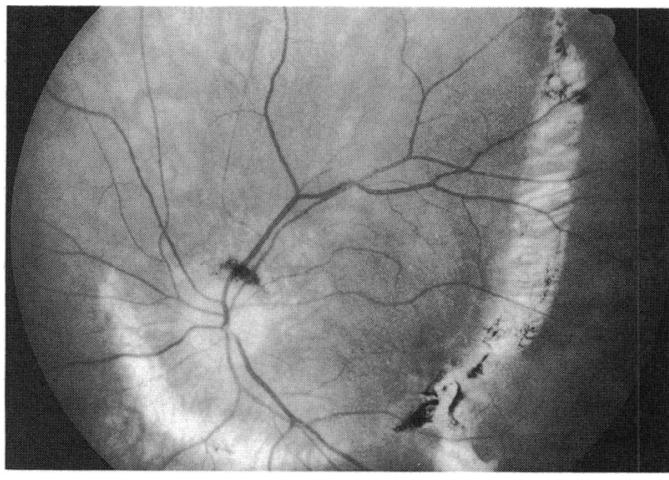

Figure 4: Fundal photograph of indirect choroidal ruptures following severe ocular trauma due to repeated kicks in the face during an assault.
TABLE III The final visual acuity related to the site of the choroidal rupture

\begin{tabular}{lllll}
\hline & $\begin{array}{l}\text { Temporal } \\
(n=13)\end{array}$ & $\begin{array}{l}\text { Nasal } \\
(n=4)\end{array}$ & $\begin{array}{l}\text { Inferior } \\
(n=8)\end{array}$ & $\begin{array}{l}\text { Superior } \\
(n=5)\end{array}$ \\
\hline $6 / 5-6 / 12$ & 5 & 4 & 6 & 2 \\
$6 / 18-6 / 60$ & 3 & - & 2 & 1 \\
$<6 / 60$ & 5 & - & - & 2 \\
\hline
\end{tabular}

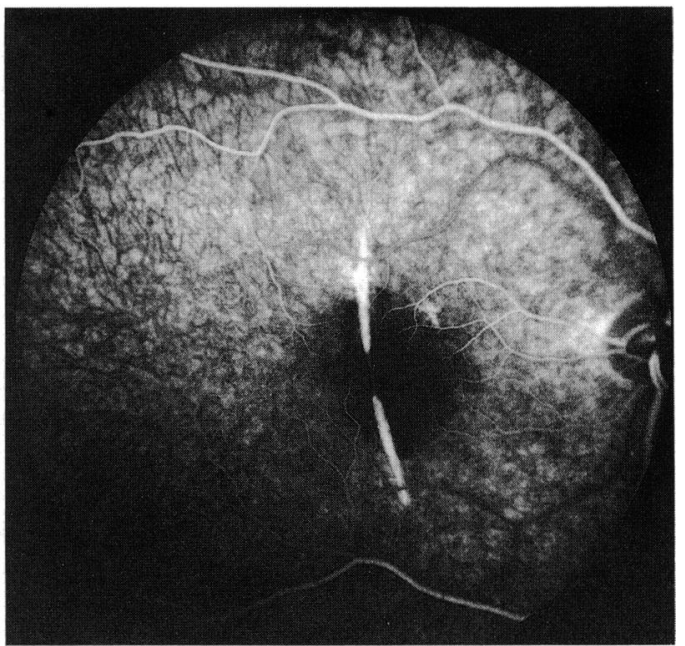

Figure 5: Fluorescein angiogram of a smooth curved rupture through the perifoveal area in an eye regaining $6 / 5$ vision after the injury, though late visual loss occurred due to a disciform maculopathy.

choroid, leading to ruptures or tears at these sites. This predicted pattern of damage is in keeping with our clinical findings.

Punch injuries have a diffuse impact on the globe, owing to the protective effect of the orbital margin, and the ruptures are exclusively peripapillary. This represents damage occurring only at the most vulnerable site, whereas injuries due to a projectile which has a focal, unimpeded impact on the globe result in more widespread ocular damage due to the higher energy imparted. The great majority of indirect choroidal ruptures occur temporally or inferiorly to the disc, reflecting vulnerability of the eye to injury from the inferotemporal direction ${ }^{9}$ rather than localised differences in Bruch's membrane or the choroid.

\section{CONCLUSIONS}

(1) Severe concussional eye injuries occur predominantly in young males. Of particular note is the finding that such injuries in teenagers are most likely to occur as a result of sports injuries, and this conclusion has been confirmed by a recent survey of ocular injuries. ${ }^{12}$

(2) Indirect choroidal ruptures occurring temporal to the disc are much more likely to be associated with visual loss than those occurring nasally, owing to the increased risk of macular damage from the direct effect of the rupture,

TABLE IV Final visual acuity according to the type of injury punches (diffuse impact on the globe), or projectile/weapon (focal impact on the globe)

\begin{tabular}{llll}
\hline & $\begin{array}{l}\text { Punches } \\
(n=7)\end{array}$ & $\begin{array}{l}\text { Projectiles } \\
(n=23)\end{array}$ & $\begin{array}{l}\text { Overall } \\
(n=30)\end{array}$ \\
\hline $6 / 5-6 / 12$ & 5 & 12 & 17 \\
$6 / 18-6 / 60$ & 1 & 5 & 6 \\
$<6 / 60$ & 1 & 6 & 7 \\
\hline
\end{tabular}


inner retinal damage, pigmentary retinopathy, or the late development of a subretinal neovascular membrane.

(3) Peripapillary choroidal ruptures occurring in the presence of minimal anterior segment injury imply an injuring force of diffuse impact or only moderate severity. Consequently, provided the macula has not been severely damaged, the patient has a relatively good visual prognosis.

Extensive multiple ruptures which are irregular and associated with significant anterior segment damage imply that the globe has been subjected to severe deforming forces, probably of focal impact. Ocular damage is more widespread, macular and optic nerve injury more likely, with a poorer visual prognosis.

(4) Indirect choroidal ruptures are due to the generation of maximal stress at the peripapillary area in closed ocular injuries, probably mainly owing to forces generated in the wall of a gelcontaining flexible compressible sphere with external and internal fixed attachments. The peripapillary region is thus vulnerable to closed ocular trauma, and it is feasible that subclinical peripapillary ocular damage may result from repeated minor ocular trauma. The latter may play a part in the onset of maculopathies of presumed non-traumatic character.
The authors would like to thank Dr Keith Mitchell from the Department of Medical Physics at the Royal Victoria Infirmary, Newcastle upon Tyne, for his help with the discussion, Michelle Gillan and Amanda Tidswell for their secretarial help, Michael Strachan and Robert Etherington for their photographic assistance, and the consultant ophthalmologists from Sunderland and Newcastle upon Tyne who referred patients to us for the study.

1 Duke-Elder S. System of ophthalmology. Mechanical injuries. Kimpton, London: 1972: 14(1): 151-60

2 Archer DB, Canavan YM. Contusional eye injuries: retinal and choroidal lesions. Aust $\mathcal{F}$ Ophthalmol 1983; 11 : 251-64.

3 Dean Hart JC, Natsikos VE, Raistrick R, Doran RML. Indirect choroidal tears at the posterior pole: a fluorescein angiographic and perimetric study. Br f Ophthalmol 1980; 64:59-67.

4 Aguilar JP, Green RW. Choroidal rupture. A histopathological study of 47 cases. Retina $1984 ; 4: 269-75$.

5 Koval R, Teller J, Belkin M, Romem M, Yanko L, Savir H. The Israeli ocular injuries study. Arch Ophthalmol 1988; 106: 776-80.

6 Godfredsen E. Re-examination of central ruptures of the choroid. Acta Ophthalmol (Kbh) 1942; 20: 337-50.

7 Wolter JR. Coup contrecoup mechanism of ocular injuries. Am 7 Ophthalmol 1963; 56: 785-96.

8 Delori F, Pomerantzeff O, Cox MS. Deformation of the globe under high speed impact: its relation to contusion injuries. Invest Ophthalmol Vis Sci 1969; 8: 290-301.

9 Wiesenthal DT. Experimental ocular contusion. Arch Ophthalmol 1964; 71: 77-81.

10 Blight R, Dean Hart JC. Structural changes in outer retinal layers following blunt mechanical non-perforating trauma to the globe: an experimental study. BrF Ophthalmol 1977; 61: 573-87.

11 Schepens CL. In: Freeman HM, ed. Ocular trauma. New York: Appleton Century Crofts, 1979; chapter 29: 273-84.

12 Tielsch JM, Parver L, Shankar B. Time trends in the incidence of hospitalized ocular trauma. Arch Ophthalmol 1989; 107: 519-23. 